\title{
Evaluación del Estado Psicobiotipológico en Bomberos: Parámetros de Salud y Recursos Anti Estrés
}

\author{
Assessment of Biopsycho Typological State of Firefighters: \\ Health Parameters and Anti Stress Resources
}

Curilem, G. C.*; Almagià, F. A..; Yuing, F. T.* \& Rodríguez, R. F.*

CURILEM, G. C; ALMAGIÀ, F. A; YUING, F. T \& RODRÍGUEZ, R. F. Evaluación del estado psicobiotipológico en bomberos: parámetros de salud y recursos anti estrés. Int. J. Morphol., 32(2):709-714, 2014.

RESUMEN: La morfoestructura y características psicológicas de los bomberos cobran vital importancia, ya que deben poseer un rendimiento físico y psicológico de alto nivel, para afrontar los requerimientos propios de su labor. Es fundamental conocer el Biotipo, así como también, la personalidad resistente y el nivel de Síndrome Burnout presente en esta población, evaluando el perfil de voluntarios activos de las comunas de Valparaíso y Viña del Mar. Se estudiaron 101 voluntarios de sexo masculino, con edades entre los 20 y 50 años, de las compañías de Valparaíso y Viña del Mar. Se aplicó el método de somatotipo de Heath - Carter, personalidad resistente y el nivel de Síndrome Burnout guiados por la metodología de Moreno Jiménez. Los resultados indican un Somatotipo predo-

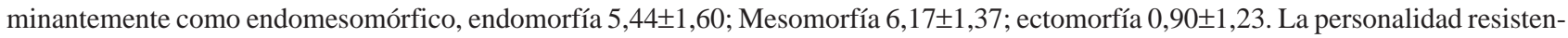

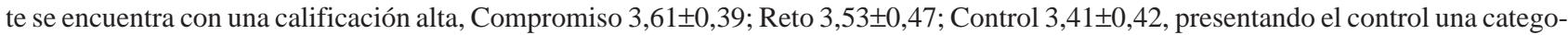

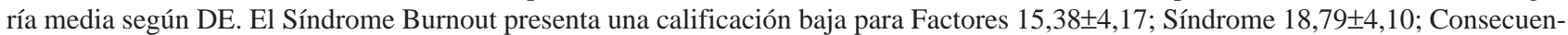
cias 5,64 $\pm 1,98$. Se destaca un alto componente Endomórfico, lo que traería consigo un riesgo cardiovascular. El perímetro de pierna está relacionado inversamente con la dimensión Control de Personalidad resistente, lo que indica que a mayor perímetro menor puntuación en control, disminuyendo la resistencia al Síndrome Burnout. Esta población no deportista, voluntaria y altruista, sin una actividad física programada, constante y periodizada, se ve reflejada en sus características antropométricas.

\section{PALABRAS CLAVE: Antropometría; Bomberos; Personalidad resistente; Síndrome Burnout.}

\section{INTRODUCCIÓN}

Profesiones de alto riesgo, son aquellas que se desarrollan en ambientes en los cuales existen o se realizan condiciones o prácticas capaces de causar incapacidad permanente, pérdida de la vida o partes del cuerpo, o pérdidas considerables de estructuras, equipos o materiales. Bomberos de Chile, en cada una de sus áreas de combates tales como: fuego, materiales peligrosos, rescate, entre otras, se encuentra constantemente en situaciones y ambientes de alto riesgo. La tarea del bombero, es una labor que somete al hombre a una extenuante y constante exigencia de sus características físicas, como también psicológicas. Por tanto, el biotipo debe ser adecuado, para cumplir con tan benéfica labor y soportar fatigosas horas, sin un horario predeterminado (Moreno Jimenez et al., 2006; Almagià et al., 2009). El estrés mental al que son sometidos alcanza los límites humanos, por no disponer de un horario fijo de trabajo, no recibir remuneración por su labor, tener que cumplir turnos, además de su trabajo fijo, tener que lidiar con el sufrimiento de las víctimas que son rescatadas y la desolación del resultado de las catástrofes. Algunas psicopatologías presentes en bomberos han comenzado a ser estudiadas hace algunos años en otros países. Principalmente se han realizado estudios sobre el cambio de horario presente en algunas profesiones, la carga de trabajo, el estado de alerta permanente y las situaciones impactantes o traumáticas a las que están enfrentados. Los estudios muestran que el cambio de horario, y por ende del ciclo sueño - vigilia, producen alteraciones psicológicas, pudiendo finalmente significar generación de psicopatologías como depresión e incluso rasgos psicopáticos.

El estrés es una de las variables más estudiadas en psicología deportiva. Es una reacción del organismo ante situaciones que le resultan amenazantes, puede ser beneficioso, en pequeñas dosis, si se maneja correctamente, pero es perjudicial cuando es excesivo y no se dispone de habi- 
lidades para controlarlo. El Síndrome Burnout es una respuesta al estrés laboral crónico, que conlleva la vivencia de encontrarse emocionalmente agotado, el desarrollo de actitudes y sentimientos negativos hacia las personas con las que se trabaja y la aparición de procesos de devaluación del propio rol profesional. Entre las características que puede presentar el Síndrome Burnout se incluye fatiga, desgaste emocional, incapacidad de concentración, ansiedad, insomnio, irritabilidad, incremento del consumo de alcohol o drogas y depresión; demás, de la vivencia de encontrarse emocionalmente agotado, desarrollo de actitudes y sentimientos negativos hacia las personas con las que se trabaja y la aparición de procesos de disminución del rol profesional. Las variables de personalidad positiva, una de las más conocidas es el constructo de Personalidad Resistente, ha sido ampliamente utilizado para comprender los procesos vinculados a la salud - enfermedad. Los individuos con personalidad resistente se enfrentan de forma activa y comprometida a los estímulos estresantes, percibiéndolos como menos amenazantes (Franco, 2009; Garcia-Izquierdo et al., 2006, 2009; Moreno Jimenez et al., 2008; Larumbe Zabala et al., 2009; Johnson et al., 2010).

La personalidad resistente surge a partir de la idea de "protección" frente a los estímulos estresantes, y no puede entenderse sino como resultado de la interacción individuo - medio, tratando de ser un concepto clave en la psicología social del estrés y la salud. Este constructo está constituido por tres dimensiones: Compromiso, control y reto. El compromiso es la tendencia a desarrollar comportamientos caracterizados por la implicación personal en todas las actividades de la vida, y es una cualidad que es propuesta como moderadora de los efectos de estrés. Además, esta cualidad no se limita a un sentimiento de competencia personal, sino que incluye igualmente un sentimiento de comunidad o corporación. El control hace referencia a la convicción que tiene el sujeto de poder intervenir en el curso de los acontecimientos. Permite al individuo percibir en muchos de los acontecimientos o situaciones estresantes, consecuencias predecibles a su propia actividad y, en consecuencia, manejar los estímulos en su propio beneficio, atenuando así los efectos del estrés. El Reto hace referencia a la creencia de que el cambio, frente a la estabilidad, es la característica habitual de la vida. Desde este punto de vista, un estímulo estresante se entendería como una oportunidad o un incentivo para el desarrollo personal, y no como una amenaza a la propia seguridad (Jaenes Sánchez, 2009; Jaenes et al., 2008; Serrano Gisbert et al., 2008). La personalidad resistente se asocia a menor Síndrome Burnout. El componente de la personalidad resistente denominado reto, amortigua la influencia de los estresores laborales en la ocurrencia del Síndrome
Burnout. La personalidad resistente actúa como modulador, que reduce la posibilidad de experimentar el desgaste profesional ante la presencia de estresores (Ríos Rísquez et al., 2010; Moreno-Murcia, 2008).

El propósito de la siguiente investigación fue: Valorar la personalidad resistente, medir el nivel de Síndrome de Burnout y determinar las características del somatotipo, correlacionando los datos para comprobar si existe alguna relación entre estas variables que son genotípica y fenotípicamente constituidas.

\section{SUJETOS Y MÉTODO}

Se consideró la participación de 101 Bomberos voluntarios evaluados, de sexo masculino, edad media $32,4 \pm 12,4$ años, previo consentimiento firmado para realizar las evaluaciones, todos los sujetos pertenecientes a distintas compañías de Bomberos de Valparaíso y Viña del Mar.

Este estudio es de tipo no experimental, descriptivo, de diseño transversal, correlacionar con un muestreo no probabilístico, presenta las variables Personalidad Resistente, Síndrome Burnout y somatotipo; la metodología usada fue: Cuestionario breve de Burnout, Cuestionario de personalidad pesistente (Moreno Jimenez et al,. 2006) y Heath \& Carter para Somatotipo. Materiales: Kit antropométrico Roscraff, Canadá; Caliper Slimguide; Pesa electrónica Gama, modelo HCB. Capacidad 150 $\pm 0,1$ kg; además Software SPSS y Minitab.

\section{RESULTADOS}

La media de bomberos fue: para edad 32,4 $\pm 12,4$ años; la estatura $173,48 \pm 7,92 \mathrm{~cm}$; el peso $85,45 \pm 14,77 \mathrm{~kg}$; El IMC fue: $28,35 \pm 4,64$. El somatotipo promedio de los bomberos fue: endomorfía $5,44 \pm 1,60$; mesomorfía $6,17 \pm 1,37$; ectomorfía $0,90 \pm 1,23$.

Los puntajes obtenidos en las tres clasificaciones de personalidad resistente, además de los puntajes obtenidos en las tres clasificaciones del Síndrome Burnout (Tabla I). A efectos de diagnóstico de Síndrome Burnout, se considera el punto de corte 25 puntos. Es decir, a partir de 25 puede considerarse que una persona está afectada claramente por el síndrome del Burnout. Al observar el coeficiente de variación $(\mathrm{CV})$ existe una homogeneidad entre las mediciones que se realizaron, exceptuando en las variables: ectomorfía, y consecuencia Burnout. 
CURILEM, G. C; ALMAGIÀ, F. A; YUING, F. T \& RODRÍGUEZ, R. F. Evaluación del estado psicobiotipológico en bomberos: parámetros de salud y recursos anti estrés. Int. J. Morphol., 32(2):709-714, 2014

Tabla I. Datos personalidad resistente, Síndrome Burnout y somatotipo bomberos.

\begin{tabular}{lccccc}
\hline $\mathbf{n}=\mathbf{1 0 1}$ & Mínimo & Máximo & Media & D.E. & CV \\
\hline Compromiso & 10 & 28 & 25,28 & 2,79 & 0,11 \\
Reto & 10 & 28 & 24,76 & 3,29 & 0,13 \\
Control & 14 & 28 & 23,89 & 2,99 & 0,13 \\
Factor Burnout & 9 & 25 & 15,38 & 4,17 & 0,27 \\
Síndrome Burnout & 9 & 30 & 18,79 & 4,10 & 0,22 \\
Consecuencias Burnout & 3 & 12 & 5,64 & 1,98 & 0,35 \\
Mesomorfía & 3,54 & 11,03 & 6,17 & 1,37 & 0,22 \\
Ectomorfía & $-1,49$ & 4,51 & 0,90 & 1,23 & 1,37 \\
\hline
\end{tabular}

Tabla II. Correlación personalidad resistente y somatotipo.

\begin{tabular}{llcccc}
\hline $\mathbf{n = 1 0 1}$ & Endomorfía & Mesomorfía & Ectomorfía & $\begin{array}{c}\text { Perímetro de } \\
\text { pierna (cm) }\end{array}$ \\
\hline Compromiso & Correlación de Pearson & 0,067 & $-0,107$ & 0,082 & $-0,109$ \\
& Significancia (bilateral) & 0,504 & 0,285 & 0,413 & 0,277 \\
\multirow{2}{*}{ Reto } & Correlación de Pearson & 0,087 & $-0,038$ & 0,003 & $-0,034$ \\
\multirow{2}{*}{ Control } & Significancia (bilateral) & 0,387 & 0,708 & 0,976 & 0,737 \\
& Correlación de Pearson & $-0,045$ & $-0,168$ & 0,045 & $-0,198$ \\
& Significancia (bilateral) & 0,656 & 0,093 & 0,652 & 0,047 \\
\hline
\end{tabular}

Tabla IIII. Correlación síndrome Burnout y somatotipo.

\begin{tabular}{llccc}
\hline $\mathbf{n = 1 0 1}$ Factor es Burnout & & Endomorfia & Mesomorfia & Ectomorfia \\
\hline \multirow{3}{*}{ Síndrome Burnout } & Correlación Pearson & 0,096 & 0,055 & $-0,106$ \\
& Significancia (bilateral) & 0,340 & 0,583 & 0,291 \\
Consecuencias Burnout & Correlación Pearson & 0,000 & 0,044 & $-0,018$ \\
& Significancia (bilateral) & 0,997 & 0,665 & 0,860 \\
& Correlación Pearson & 0,094 & $-0,061$ & 0,002 \\
& Significancia (bilateral) & 0,350 & 0,545 & 0,981 \\
\hline
\end{tabular}

La Figura 1, muestra los datos estadísticos de los somatotipos individuales de Bomberos en la carta Somática. La nube de somatotipos se distribuye mayormente en los segmentos de mesomorfía - endomorfía. El somatotipo promedio se ubica en el límite entre la mesomorfía y endomorfía, alejado del centro de la somatocarta, indicando una adiposidad relativa aumentada y una alta robustez músculo esquelética; con baja linealidad, apariencia física redondeada.

La variable control solo se encuentra correlacionada un $-0,198$ (p:0,05) con la variable perímetro de pierna, indicando que a mayor perímetro de pierna menor será el puntaje obtenido en la clasificación control de personalidad persistente (Tablas II y III), indicando que a mayor perímetro menor puntaje en xontrol, reduciendo la resistencia al síndrome Burnout. La variable factores de Burnout, se encuentran inversamente correlacionadas con todas las variables pertenecientes en la personalidad persistente.

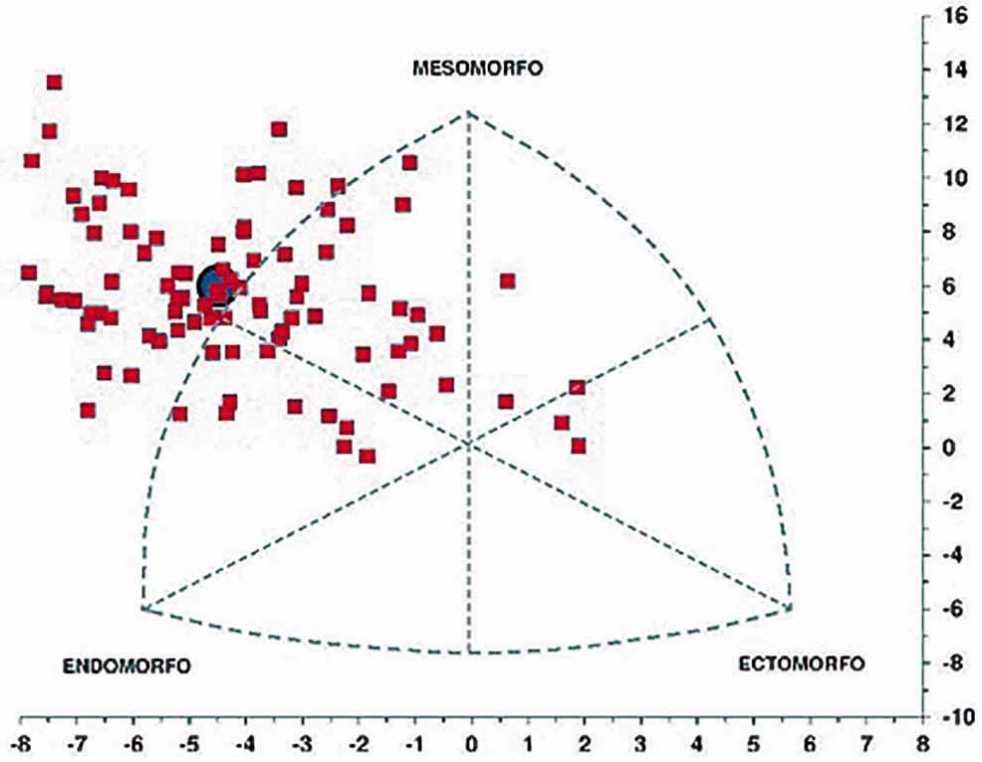

Fig. 1. Somatotipo promedio y dispersión de los Somatotipos individuales $(\mathrm{n}=101)$. 


\section{DISCUSIÓN}

Los bomberos están expuestos a sobreesfuerzos, trastornos musculo esqueléticos y alteraciones del equilibrio postural. Además, el equipamiento que ocupan, traje, equipo de respiración autónomo, herramienta de trabajo, entre otras, puede llegar a pesar entre 20 a 30 kilos. Por lo tanto su biotipo debe poseer una masa muscular desarrollada para estar acorde, asimismo está musculatura debe tener un alto rendimiento en fuerza y resistencia, para evitar estas lesiones. El biotipo se convierte en un elemento esencial para la realización de su labor (Da Silva, 2011; Rodriguez et al., 2010; Fernández et al., 2008; Pailhez et al., 2009). Una vez equipado debe transportar victimas, levantar mangueras, trasladar muebles o equipos al lugar del siniestro. Por lo cual, sus requerimientos físicos aumentan considerablemente. Adicionalmente, por la geografía de la Región de Valparaíso, deben trasladarse a cerros, espacios confinados, edificios, terrenos con pendientes, laderas, entre otras. Otra de las tareas que requiere gran energía es atender accidentes de tráfico, donde se realiza extricación de personas atrapadas. Para realizar esta actividad se utilizan herramientas hidráulicas, de gran peso y que necesitan gran fuerza para ejercer su labor y poder rescatar a las víctimas (Rios Risquez et al., 2011; Perez Hidalgo \& Rodriguez Gonzalez, 2011).

El propósito de esta investigación fue analizar las relaciones existentes entre el Síndrome Burnout, la personalidad resistente y el somatotipo. Tomando como referencia el marco teórico previo, podemos concluir que los resultados obtenidos confirman los hallazgos encontrados por otros autores (Moreno Jimenez et al., 2006). En este sentido, los análisis realizados ponen de manifiesto la existencia de una relación inversamente significativa entre las dimensiones que constituyen el Síndrome de Burnout y una mayor personalidad resistente, encontrada en esta muestra. Además, los resultados también reflejan que existe una asociación negativa significativa entre la variable control de personalidad resistente y el perímetro de pierna en los parámetros de medición del aomatotipo. Esto puede tener relación con el hecho que a mayor perímetro de pierna se posee una masa corporal mayor, que en esta muestra se relaciona con un componente endomórfico aumentado; por tanto, presentar una adiposidad mayor disminuye el "control" sobre las situaciones a las que se ven enfrentados en su labor; Así también, poseer una dimensión de "Control" disminuida aumenta la posibilidad presentar Síndrome de Burnout (Garcia-Izquierdo et al., 2009; Miró et al., 2007; Jaenes Sánchez). Con respecto a la relación entre personalidad resistente y Síndrome Burnout, podemos concluir que, estos dos constructos psicológicos se encuentran negativamente relacionados de forma significativa, hay que destacar la especial asociación inversa de la variable Factores de Burnout, y las dimensiones de la personalidad resistente. Además, la variable consecuencias de Burnout se encuentra asociada inversamente con la variable control perteneciente a la personalidad resistente. Estos hallazgos resultan en parte congruentes con los encontrados por estudios realizados en personal de enfermería, medicina, bomberos y atletas (Garmendia, 2007; Jiménez, 2008; Merino et al., 2007; Johnson; Larumbe Zabala et al., 2009; Carvalho et al., 2006; Rios Risquez et al., 2010, 2011).

Con respecto a la biotipología, el margen de peso apropiado, según la estatura promedio, debería encontrarse entre los 60-75 kilos; en la muestra de Bomberos el promedio de peso se encuentra sobre el límite superior. El índice de masa corporal indica sobrepeso para la población Chilena, indicando un riesgo de morbimortalidad levemente aumentado, presentando compromiso de sufrir un evento cardiovascular fatal. El somatotipo que presentan los bomberos se caracteriza como endomesomórfico, destacando el componente Endomórfico en alta puntuación, siendo un lastre para realizar la actividad bomberil y de rescate. (Fernández et al.; Rodriguez et al.). El componente Mesormórfico presenta una calificación Alta (Da Silva), debido a la actividad física que realizan los bomberos, generando actividades de fuerza - resistencia, este componente es imprescindible en actividades de rescate. El componente ectomórfico presenta una calificación baja, esté debería aumentar disminuyendo el componente endomórfico, que en este tipo de actividad no genera ningún beneficio, aumenta el riesgo de enfermedades crónicas no transmisibles, disminuyendo la capacidad de realizar labores de rescate de manera eficaz y seguras. Además, incrementa el riesgo de presentar eventos cardiovasculares agudos con resultado fatal. Este tipo de población es no deportista, sin una actividad física programada y constante, lo que se demuestra en sus características antropométricas, además los tipo de alimentación, actividad física y estilos de vida son similares a la sociedad actual chilena, con los estándares reflejados en la última encuesta de salud. Un buen control nutricional y actividad física dirigida adecuadamente por un equipo multidisciplinario son necesarios para estos voluntarios que entregan su vida por salvar otras de forma altruista y desinteresada.

Con la presente investigación se ha pretendido profundizar en la relevancia que determinadas variables de tipo individual o personal pueden tener a la hora de explicar el estado psicológico y físico de Personal de Bomberos. Además, evaluar los parámetros de salud de estas dos dimensiones, referidas en la definición de salud de la OMS «La salud es un estado de completo bienestar físico, mental y social, y no solamente la ausencia de afecciones o enfermedades.». 
A pesar de que el constructo de personalidad resistente se originó hace ya más de tres décadas, en nuestro contexto sociocultural más próximo resultan escasos los estudios que han investigado esta importante variable individual protectora ante los efectos del estrés en el personal de Bomberos (Moreno Jimenez et al., 2008). Además, no encontramos estudios que evalúen el Somatotipo relacionándolo con una variable psicológica en este tipo de población en nuestro país (Almagià et al.; Jaenes et al.).

Finalmente, nuestro estudio presenta ciertas limitaciones que es preciso matizar. En primer lugar, analizamos una muestra de profesionales seleccionada de forma no aleatoria y que han participado de forma voluntaria en el estudio. Además, un mayor tamaño de muestra y procedente de diferentes regiones hubiera permitido una mayor generalización de los resultados obtenidos. Futuras investigaciones deberían utili- zar diseños y análisis más complejos, con el fin de poder esclarecer mejor el papel desempeñado por el constructo de la Personalidad resistente en Síndrome Burnout, y estado Biotipológico de este tipo de poblaciones.

\section{CONCLUSIONES}

El somatotipo medio de la muestra es endomesomórfico. Las dimensiones de personalidad resistente están relacionadas inversamente con el Síndrome Burnout. La dimensión control se encuentra inversamente relacionada con el perímetro de pierna, lo que nos permite inferir que a mayor masa corporal, aumentada por un componente Endomórfico alto, disminuye la Resistencia frente al desarrollo del Síndrome Burnout.

CURILEM, G. C; ALMAGIÀ, F. A; YUING, F. T; RODRÍGUEZ, R. F. Assessment of biopsycho typological state of firefighters: Health parameters and anti stress resources. Int. J. Morphol., 32(2):709-714, 2014.

SUMMARY: The morphostructural analysis and psychological characteristics of firefighters are vital; specific physical and psychological levels are needed to meet the requirements of their work. It is essential to know biotype, personality resistance variables and level of burnout syndrome present in this population. We evaluated the profile of active volunteers in the cities of Valparaiso and Viña del Mar in Chile; 101 male volunteers, aged between 20 and 50 years were included in the study. Heath - Carter somatotype, Personality Resistance variables and level of Burnout Syndrome according to Jiménez Moreno methodology were applied. The results

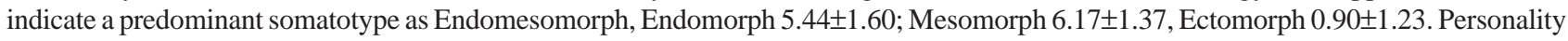
resistance variable presented a high score, commitment 3.61 \pm 0.39 , challenge $3.53 \pm 0.47$, control 3.41 \pm 0.42 . Burnout syndrome had a low factor rating $15.38 \pm 4.17$, syndrome $18.79 \pm 4.10$; consequences $5.64 \pm 1.98$. There is a high endomorphic component increasing cardiovascular risk. Leg circumference is on the other hand, related to Personality resistance control, indicating that while perimeter is greater, the mark is lower for Burnout resistance syndrome. The lack of continuous and programmed physical activity in this population of volunteer firefighters is clearly reflected in their anthropometric characteristics.

KEY WORDS: Anthropometry; Firefighters; Resistant Personality; Burnout.

\section{REFERENCIAS BIBLIOGRÁFICAS}

Almagià, F. A.; Lizana, A. P.; Rodriguez, R. F.; Ivanovic, M. D. \& Binvignat, G. O. Variables antropometricas y rendimiento fisico en estudiantes universitarios de educacion fisica. Int. J. Morphol., 27(4):971-5, 2009.

Carvalho, V. M. L.; Calvo, B. F.; Martin, L. H.; Campos, F. R. \& Castillo, I. C. Resiliencia y el modelo Burnout-Engagement en cuidadores formales de ancianos. Psicothema, 18(4):7916, 2006.

Da Silva, A. Somatotipo y aptitud fisica en arbitros asistentes de futbol. Int. J. Morphol., 29(3):792-8, 2011.

Fernández, V.; Da Silva, A. \& Arruda, M. Perfil antropometrico y aptitud fisica de arbitros de futbol profesional Chileno. Int. J. Morphol., 26(4):897-904, 2008.
Franco, C. Modificación de los niveles de Burnout y de personalidad resistente en un grupo de deportistas a través de un programa de conciencia plena (mindfulness). Anu. Psicol., 40(3):377-90, 2009.

Garcia-Izquierdo, A. L.; Ramos Villagrasa, P. J. \& Garcia Izquierdo, M. Los Big Five y el efecto moderador de la resistencia en el agotamiento emocional. Rev. Psicol. Trab. Organ., 25(2):135-47, 2009.

Garcia-Izquierdo, M.; Llor Esteban, B.; Leon Garcia-Izquierdo, A. \& Ruiz Hernandez, J. A. Bienestar psicológico y mobbing en una muestra de profesionales de los sectores educativos y sanitario. Rev. Psicol. Trab. Organ., 22(3):381-95, 2006.

Garmendia, M. L. Análisis factorial: una aplicación en el cuestio- 
nario de salud general de Goldberg, version de 12 preguntas. Rev. Chil. Salud Pública, 11(2):57-65, 2007.

Jaenes Sánchez, J. C. Personalidad resistente en deportes. Rev. Andal. Med. Deporte, 2(3):98-101, 2009.

Jaenes, J. C.; Godoy, D. \& Román, F. J. Elaboración y validación psicometrica de la escala de personalidad resistente en maratonianos (EPRM). Cuad. Psicol. Deporte, 8(2):59-81, 2008.

Jiménez, M. Bienestar psicológico y habitos saludables: ¿están asociados a la practica de ejercicio físico? Int. J. Clin. Health Psychol., 8(1):185-202, 2008.

Johnson, B. T.; Scott-Sheldon, L. A. \& Carey, M. P. Meta-synthesis of health behavior change meta-analyses. Am. J. Public Health, 100(11):2193-8, 2010.

Larumbe Zabala, E.; Perez-Llantada, R. C. \& Lopez de la Llave, R. A. Características del estado psicológico de los corredores populares de maraton. Rev. Psicol. Deporte, 18(2):151-63, 2009.

Merino, C.; Manrique, G.; Angulo, M. \& Isla, N. Indicador de estrategias de afronatmiento al estres: analisis normativo y de su estructura factorial. Ansiedad y Estrés, 13(1):25-40, 2007.

Miró, E.; Solanes, A.; Martinez, P.; Sanchez, A. \& Rodriguez, J. Relación entre el Burnout o "síndrome de quemarse por el trabajo", la tensión laboral y las características del sueño. Psicothema, 19(3):388-94, 2007.

Moreno Jimenez, B.; Morante, M.; Rodriguez, R. \& Rodriguez, A. Resistencia y vulnerabilidad en el trauma: el efecto moderador de las variables de personalidad. Psicothema, 20(1):12430,2008

Moreno Jimenez, B.; Morett, N.; Rodriguez, A. \& Morantes, M. La personalidad resistente como variable moduladora del sindrome burnout en una muestra de bomberos. Psicothema, 18(3):413-8, 2006

Moreno-Murcia, J. Motivación y salud en la practica fisico-deportiva: diferencias según el consumo de alcohol y tabaco. Int. J. Clin. Health Psychol., 8(2):483-94, 2008.

Pailhez, G.; Rodriguez, A.; Ariza, J.; Palomo, A. \& Bulbena, A. Somatotipo y ezquizofrenia. un estudio de caso-control. Actas Esp. Psiquiatr., 37(5):258-66, 2009.

Perez Hidalgo, A. \& Rodriguez Gonzalez, J. Analisis del estado emocional de una unidad del ejercito español en zona de operaciones. Sanid. Mil., 67(2):71-7, 2011.

Rios Risquez, M.; Godoy Fernandez, C. \& Sanchez-Meca, J. Síndrome de quemarse por el trabajo, personalidad resistente y malestar psicológico en personal de enfermería. Anal. Psicol., 27(1):71-9, 2011.

\section{4}

Ríos Rísquez, M.; Sanchez Meca, J. \& Godoy Fernandez, C. Personalidad resistente, autoeficacia y estado general de salud en profesionales de enfermeria de cuidados intensivos y urgencias. Psicothema, 22(4):600-5, 2010.

Rodriguez, R. F.; Almagià, A.; Yuing, T.; Binvignat, O. \& Lizana, P. Composición corporal y somatotipo referencial de sujetos fisicamente activos. Int. J. Morphol., 28(4):1159-65, 2010.

Serrano Gisbert, M.; Garces de los Fayos, E. \& Hidalgo Montecinos, M. Burnout en fisioterapeutas españoles. Psicothema, 20(3):361-8, 2008

Dirección para correspondencia:

Atilio Aldo Almagià Flores

Laboratorio de Antropología Física y Anatomía Humana

Instituto de Biología, Facultad de Ciencias

Avenida Universidad 330

Curauma, Placilla

Pontificia Universidad Católica de Valparaíso

Valparaíso

CHILE

Email: aalmagia@gmail.com; aalmagia@ucv.cl

Recibido : 17-11-2013

Aceptado: $26-02-2014$ 